\title{
The Development of an Amperometric Enzyme Biosensor Based on a Polyaniline-Multiwalled Carbon Nanocomposite for the Detection of a Chemotherapeutic Agent in Serum Samples from Patients
}

\author{
Ahmad Gholami $\mathbb{D}^{1,2}$ Fatemeh Farjami ${ }^{1}{ }^{1},{ }^{1}$ and Younes Ghasemi $\mathbb{D}^{1,2}$ \\ ${ }^{1}$ Pharmaceutical Sciences Research Centre, Shiraz University of Medical Sciences, Shiraz, Iran \\ ${ }^{2}$ Biotechnology Research Centre, Shiraz University of Medical Sciences, Shiraz, Iran \\ Correspondence should be addressed to Fatemeh Farjami; fatemehfarjami@gmail.com
}

Received 24 February 2021; Revised 5 May 2021; Accepted 30 June 2021; Published 14 July 2021

Academic Editor: J.G. Manjunatha

Copyright (c) 2021 Ahmad Gholami et al. This is an open access article distributed under the Creative Commons Attribution License, which permits unrestricted use, distribution, and reproduction in any medium, provided the original work is properly cited.

\begin{abstract}
Polyaniline was electrochemically polymerized onto the platinum electrode modified with a mixture of Nafion and multiwalled carbon nanotube (PANI/MWCNT/Nf/Pt) to detect ammonium ion. The nanobiosensor (ASNase/PANI/MWCNT/Nf/Pt) was then prepared by immobilizing L-asparaginase (L-ASNase) on the PANI/MWCNT/Nf nanocomposite. The prepared nanobiosensor was used for the rapid and sensitive detection of serum concentration of the anticancer agent L-asparagine (LAsp) during chemotherapy. The nanobiosensor has dynamic ranges of zero to $180 \mu \mathrm{M}$. The sensitivity of the nanobiosensor was $0.829 \mu \mathrm{A} \mu \mathrm{M}^{-1} \mathrm{~cm}^{-2}$, and the response time was less than $30 \mathrm{~s}$. The detection limit was $140 \mathrm{nM}$ of L-Asp. The Michaelis-Menten constant $\left(K_{m}\right)$ was measured to be $36.2 \mathrm{mM}$. The nanobiosensor was successfully applied for the determination of L-Asp in the blood samples of leukemia patients.
\end{abstract}

\section{Introduction}

L-Asparagine (L-Asp) is a nonessential amino acid synthesized by the cells on their own by asparagine synthetase. The cancer cells cannot synthesize L-Asp and rely on the extracellular supply in plasma and tissue. L-Asparaginase (L-ASNase) is a chemotherapeutic agent which can break down L-Asp into aspartic acid and ammonium ion. Due to this process, the cancer cells die as they cannot accomplish their protein synthesis. L-ASNase is extensively used for the treatment of childhood acute lymphoblastic leukemia [1].

It is critical to determine and adjust the L-Asp concentration in the patients' blood during treatment because this chemotherapeutic protein has a narrow therapeutic index. Over the last decades, some efforts have been made to develop methods for determining L-Asp in serum samples. Standard instrumental techniques such as ion-pair chromatography [2], spectrophotometry [3], and colorimetric assay [4] have been used for the determination of L-Asp; however, these methods are often sophisticated, time-consuming, and very expensive.

Enzymatic methods for determining amino acid levels are an important route in detecting several biopharmaceuticals [5]. Biosensors containing electrochemical transducers, mostly with potentiometric ones [6], have been developed based on the measurement of ammonium ions produced during the enzymatic destruction of L-Asp. L-ASNase, as a catalytic enzyme, hydrolyzes L-Asp to L-aspartic acid and $\mathrm{NH}_{4}^{+}[1,7]$. Analytical studies such as equilibrium dialysis and fluorescence quenching revealed that about one mole of L-aspartic acid and one mole of $\mathrm{NH}_{4}^{+}$were produced due to hydrolysis of one mole L-asparagine by one mole of LASNase, with a dissociation constant on the order of 60 $160 \mu \mathrm{M}$ [8]. However, the significant drawbacks of potentiometric biosensors are the slow response to a steady-state potential value and a high detection limit $[9,10]$. In contrast, 
amperometric biosensors represent better sensitivity, quicker responses, and a lower detection limit [11]. The mentioned properties make them a proper choice for the rapid and reliable tool for determining the L-Asp level in biological samples. Since $\mathrm{NH}_{4}^{+}$ions are not electroactive, for amperometric determination of this ion, systems with the application of the composite polymers of Nafion with polyaniline (PANI) or polypyrrole were used [12]. PANI is considered a fascinating conductive polymer for designing enzyme biosensors because it has great electrochemical tunability, redox actions, electrocatalytic conduction, good processability, and environmental stability, along with a flexible chemical structure with the ability to be functionalized [13]. Chowdhury et al. showed that PANI nanowires (decorated with gold nanostructures) were successfully used for biopharmaceutical sensing with advantages of low detection limit, excellent sensitivity, and long-term stability [14].

One of the most crucial characteristics of conducting polymers is their ability to reversibly alternate between the conducting and insulating electronic states by dopingundoping of ions [15-20]. Anions were shown to be the dopants in polyaniline films [21]. However, polyaniline electrodeposition on Nafion film results in a Nafion-coated polyaniline composite with a cation doping-undoping behavior [22]. Nafion is a cation exchanger conducting polymer. The Nafion-polyaniline composite could effectively sense the ammonium ion; therefore, this composite was used in enzymatic biosensors based on $\mathrm{NH}_{4}^{+}$detection like urea and L-arginine biosensors [23].

Recently, Shu and Tang reviewed the recent advances in photoelectrochemical sensing [24]. They mentioned engineering photoactive materials through photoelectric conversion, which involves kinetics and thermodynamics and conversion efficiency. Semiconductor-carbon heterojunction such as multiwalled carbon nanotubes (MWCNTs) and single-walled carbon nanotubes (SWCNTs) can improve charge separation/transfer in electrochemical biosensors $[25,26]$. The introduction of MWCNTs into the PANI matrix [27] and MWCNT into Nafion [28] and their application to construct biosensors were repeatedly reported. Different nanocomposites of Nafion, MWCNT, and polyaniline were constructed [29-31]; however, to the best of our knowledge, they were not used to fabricate amperometric ammonium ion sensors or in the biosensors based on the detection of ammonium ions.

In this work, the nanocomposite of PANI/MWCNT/Nafion was prepared. L-ASNase was immobilized directly on the nanocomposite, and a very sensitive L-Asp biosensor was formed. This study may open a new way for effective monitoring of L-Asp in the serum of cancer patients receiving all forms of clinically used L-ASNase, which helps physicians effectively adjust the dose of this chemotherapeutic agent.

\section{Experiment}

2.1. Reagents. Sodium hydrogen phosphate and sodium dihydrogen phosphate were purchased from Acrose. LAsparagine and ammonium chloride were bought from
Merck. L-Asparaginase was from Kyowa (Japan). Bovine serum albumin (BSA), D-homocysteic acid, 5-sulfosalicylic acid, MWCNT ( $>90 \%$ carbon basis, $D \times L$ 110-170 $\mathrm{nm} \times 5-9 \mu \mathrm{m})$, and Nafion were obtained from Sigma-Aldrich.

2.2. Apparatus. A scanning electron microscope (MIRA3TESCAN-XMU) was used to characterize the PANI/MWCNT film on the surface of a Pt electrode. FT-IR ATR spectra were recorded by using TENSOR II (Bruker, Germany). The electropolymerization of aniline was performed through a three-electrode system, including a Pt auxiliary electrode, an $\mathrm{Ag} / \mathrm{AgCl} 3 \mathrm{M} \mathrm{KCl}$ reference electrode, and a Pt disk (3.0 mm diameter) working electrode. An Autolab electrochemical system (Eco-Chemie, Utrecht, The Netherlands) equipped with Autolab PGSTAT-302N GPES software (Eco-Chemie, Utrecht, The Netherlands) was used for voltammetric experiments.

2.3. Pretreatment of MWCNT. A sample of $0.5 \mathrm{~g}$ MWCNT was suspended in $240 \mathrm{~mL}$ of concentrated nitric acid and sulphuric acid ( $1: 3$ in volume ratio) and refluxed at $80^{\circ} \mathrm{C}$ for $4 \mathrm{~h}$. After washing with copious amounts of doubly distilled water until the supernatant attained a $\mathrm{pH}$ around seven, the sample was dried in a vacuum oven at $80^{\circ} \mathrm{C}$.

2.4. Preparation of Electrodes. A $0.5 \mathrm{wt} \%$ Nafion solution was prepared by diluting a $5 \%$ sample with $90 \%$ ethanol/water. A $5 \mu \mathrm{L}$ aliquot of this solution was coated onto a working $\mathrm{Pt}$ electrode and dried in air. Then, $5 \mu \mathrm{L}$ of $0.1 \mathrm{mg} / \mathrm{mL}$ MWCNT was placed on the electrode and was dried. The MWCNT/Nf/Pt electrode was immersed in a solution containing $0.2 \mathrm{M}$ aniline in $0.5 \mathrm{M} \mathrm{HCl}$, and electropolymerization was carried out by cyclic voltammetry. The potential was swept from 200 to $1200 \mathrm{mV}$ (vs. the $\mathrm{Ag} / \mathrm{AgCl}$ reference electrode) with a scan rate of $50 \mathrm{mV} \mathrm{s}^{-1}$. The fabricated electrode (PANI/MWCNT/Nf/Pt) has a distinct light green color. The PANI/Nf/Pt electrode was made in the same way without MWCNT.

To prepare the selective membrane, a solution of $200 \mathrm{U} / \mathrm{mL}$ L-ASNase, $2 \%$ Nafion, and 2\% BSA was prepared. $5 \mu \mathrm{L}$ of this solution was drop-cast on the surface of PANI/MWCNT/Nf/Pt and allowed to dry.

2.5. Preparation of Real Samples. To study the biosensor application in real sample analysis, we tested blood samples collected from patients hospitalized in Namazi Hospital, located in Shiraz, Iran. The donor was a patient with acute lymphoblastic leukemia (ALL) who was given low doses of L-ASNase (10000 IU per $\mathrm{m}^{2}$ every 3 days). The samples were obtained $24 \mathrm{~h}$ before administration of L-ASNase and $72 \mathrm{~h}$ after the second dose.

2.6. Determination of L-Asp Concentration by the Reference Method. A total of $2 \mathrm{~mL}$ blood sample was added to $0.1 \mathrm{~mL}$ homocysteic acid solution $(100 \mu \mathrm{g} / \mathrm{mL})$ and $0.5 \mathrm{~mL}$ sulfosalicylic acid $(200 \mu \mathrm{g} / \mathrm{mL})$. The solution was vortexed and centrifuged at $4000 \mathrm{RPM}$ for $3 \mathrm{~min}$, and the supernatants were stored at $-80^{\circ} \mathrm{C}$ until further analysis. 


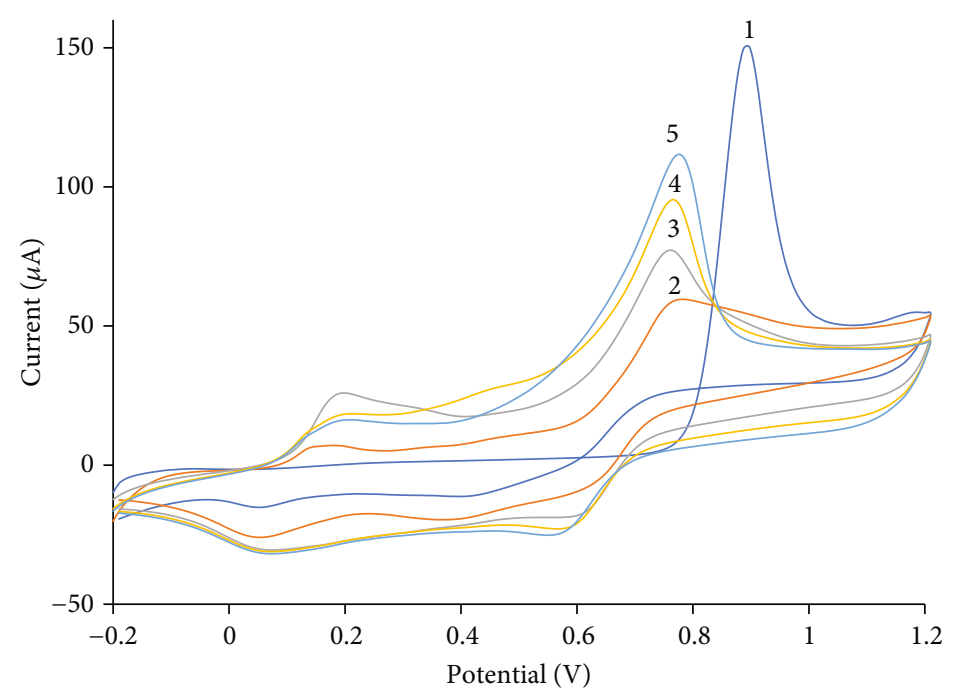

FIGURE 1: Five successive cyclic voltammograms of the Nf/Pt electrode in $0.2 \mathrm{M}$ aniline in $0.5 \mathrm{M} \mathrm{HCl}$ at a scan rate of $50 \mathrm{mV} \mathrm{s}^{-1}$.

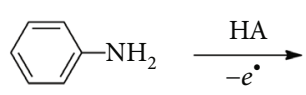<smiles>[NH3+]c1ccccc1</smiles>

$\overline{\mathrm{A}}$

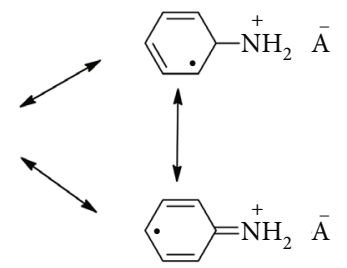

$(2)$
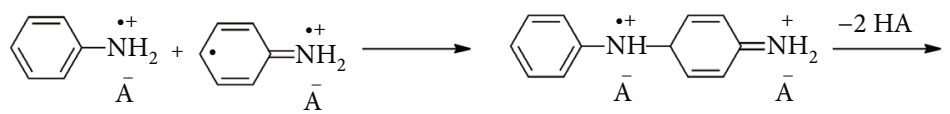<smiles>Nc1ccc(Nc2ccccc2)cc1</smiles>

(3)
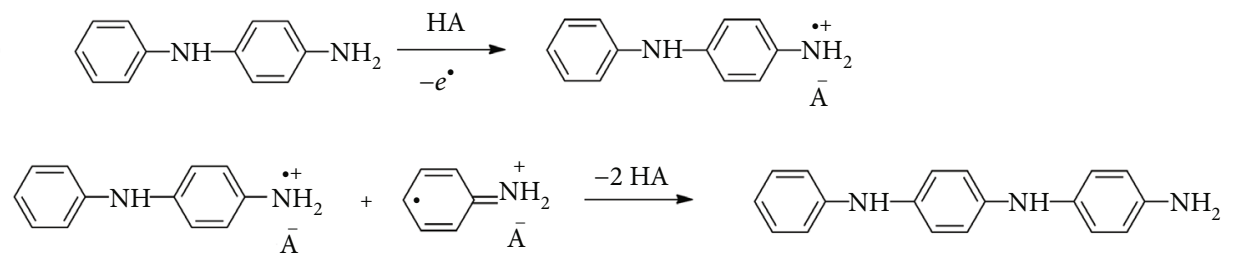

(4)

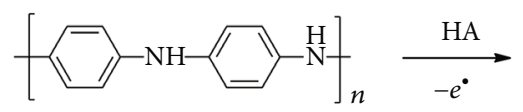<smiles>CNc1ccc(Nc2ccc(C(C)(C)C)cc2)cc1</smiles>

Basic polyaniline structure<smiles>[Y][Y](C)=NC1C=CC(=Nc2ccc(C([Y])(C)Nc3ccc(Nc4ccc(C)cc4)cc3)cc2)C=C1</smiles>

Scheme 1: The schematic diagram showing the structure and the electropolymerization mechanism of polyaniline.

The serum concentration of L-Asp was also determined using high-performance liquid chromatography (HPLC) as the reference method. The column was treated with phthaldialdehyde, and separation was done by isocratic elution with a mobile phase of water-acetonitrile-sodium propionate buffer $(82: 7.5: 10.5, v / v)$, delivered at a flow rate of $1.6 \mathrm{~mL} / \mathrm{min}$ through a Eurospher C18 column $(150 \mathrm{~mm} \times 4.6 \mathrm{~mm})$ as the stationary phase. The chromatographic pattern of L-Asp was detected by using a fluorescence detector (Knauer, Berlin, Germany) at the excitation wavelength of $360 \mathrm{~nm}$ and an emission cut-off filter of $418 \mathrm{~nm}$. The analysis of chromatograms was done by using EZChrom Elite ${ }^{\circledR}$ software. 


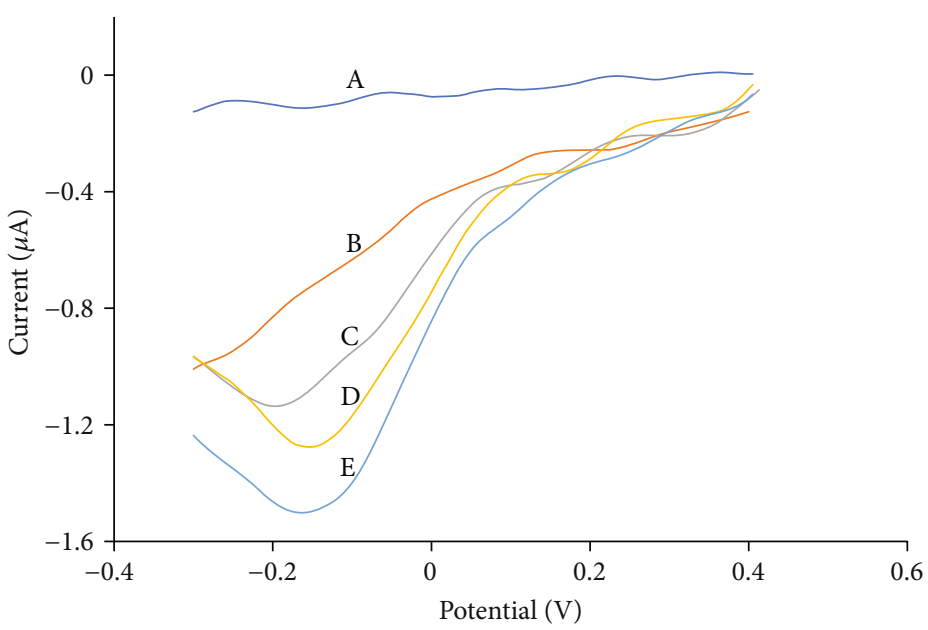

Figure 2: Linear sweep voltammograms of PANI/CNT/Nf/Pt recorded before (a) and after successive addition of $0.5 \mathrm{mM} \mathrm{NH}_{4} \mathrm{Cl}(\mathrm{b}-\mathrm{e})(\mathrm{PBS}$ $\mathrm{pH} 7$, scan rate $50 \mathrm{mV} \cdot \mathrm{s}^{-1}$ ).

\section{Results and Discussion}

As the first step for constructing the L-Asp biosensor, an ammonium sensor was developed and optimized because the proposed biosensor determines L-Asp by measuring the ammonium ion concentration produced during enzymatic destruction of L-Asp.

\subsection{Electropolymerization of Aniline on the Nf/Pt Electrode.} The CVs recorded during electrodeposition of polyaniline on the Nf/Pt electrode are shown in Figure 1. The peak positions and their intensities are analogous to those commonly observed in the literature [20]; however, they vary with the conditions of electrodeposition. The polymerization mechanism of aniline in acid medium and the PANI polymer structure is presented in Scheme 1. The CVs show that the electropolymerization processes consist of two or three electrochemical systems. The large peak at $0.9 \mathrm{~V}$ that appeared at the first scan is due to the oxidation of aniline monomer to form cation radicals (the first step of Scheme 1). The reverse sweep of the first cycle involves a crossing effect interpreted as the start of the nucleation process of the polymer. New peaks, which are ascribable to the electrochemical response of PANI deposits formed during the oxidation process of aniline, appear after the first cycle.

The following cycles indicate a normal growth of the polymeric deposit. In acidic solutions, the second step of polymerization involves an isomeric transformation of cation radicals and the formation of a dimeric intermediate product through deprotonation and rearomatization. The anodic peak around $0.1 \mathrm{~V}$ is due to the oxidation of the dimer to dimer cation radicals and then further reacts with monomer cation radicals which is the propagation step of the polymerization (step 3 of Scheme 1). Also, the oxidation of leucoemeraldin (a fully reduced form of PANI, $x=1$ ) to emeraldine (a half-reduced form of PANI, $x=0.5$ ) occurs at this potential. The cathodic peak of this redox pair is related to the reduction of emeraldine to leucoemeraldine. The redox pair around $0.7 \mathrm{~V}$ was assigned to emeraldine oxidation to pernigraniline (a fully oxidized form of PANI, $x=0$

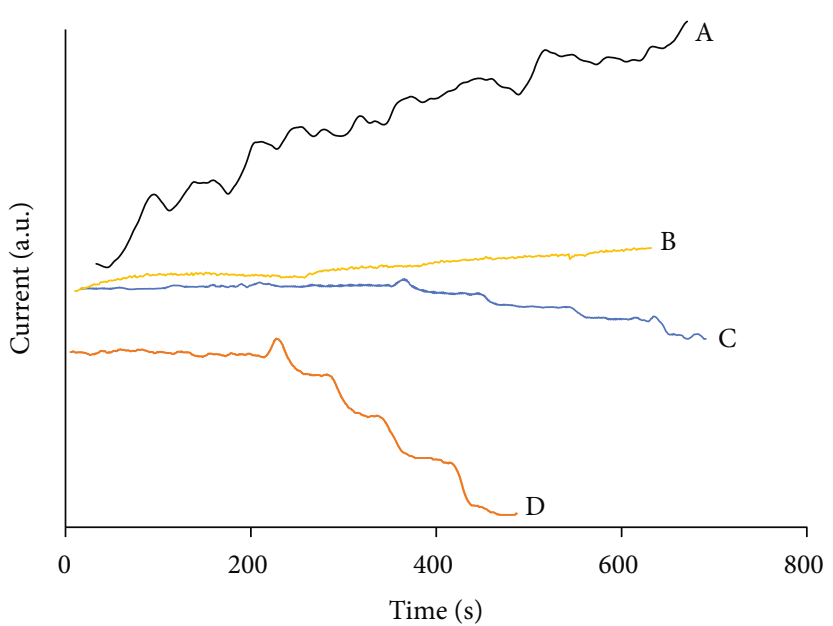

FIgURE 3: The response current-time curves of various modified electrodes Nf/Pt (a), PANI/Pt (b), PANI/Nf/Pt (c), and $\mathrm{PANI} / \mathrm{MWCNT} / \mathrm{Nf} / \mathrm{Pt}(\mathrm{d})$ at successive addition of $2.5 \mu \mathrm{M} \mathrm{NH}+$ in PBS $(0.05 \mathrm{M}, \mathrm{pH} 7)$.

) and reverse reduction reaction (step 4 of Scheme 1). The very weak peak at ca. $0.5 \mathrm{~V}$ is due to benzoquinone/hydroquinone-the products of PANI hydrolysis [21].

As the potential needed for the oxidation of the monomer is always higher than the charging of oligomeric intermediates or the resulting polymer, the two processes of polymer formation and its oxidations (doping) are simultaneously occurring. The anions are doped during electrochemical polymerization, fulfilling the request of electroneutrality. When Nafion, which is a cation exchanger, is used as a substrate, the sulfonate groups attached to the polymer backbone play the role of anions, and upon oxidation, the cationic PA polymer forms within the Nafion matrix with the expulsion of $\mathrm{Na}^{+}$; therefore, the reduction process needs cations.

$$
\mathrm{C}^{+}+\mathrm{PA}^{+} \cdot \mathrm{RSO}_{3}{ }^{-}+e^{-} \longrightarrow \mathrm{PA} \cdot \mathrm{C}^{+} \cdot \mathrm{RSO}_{3}{ }^{-}
$$




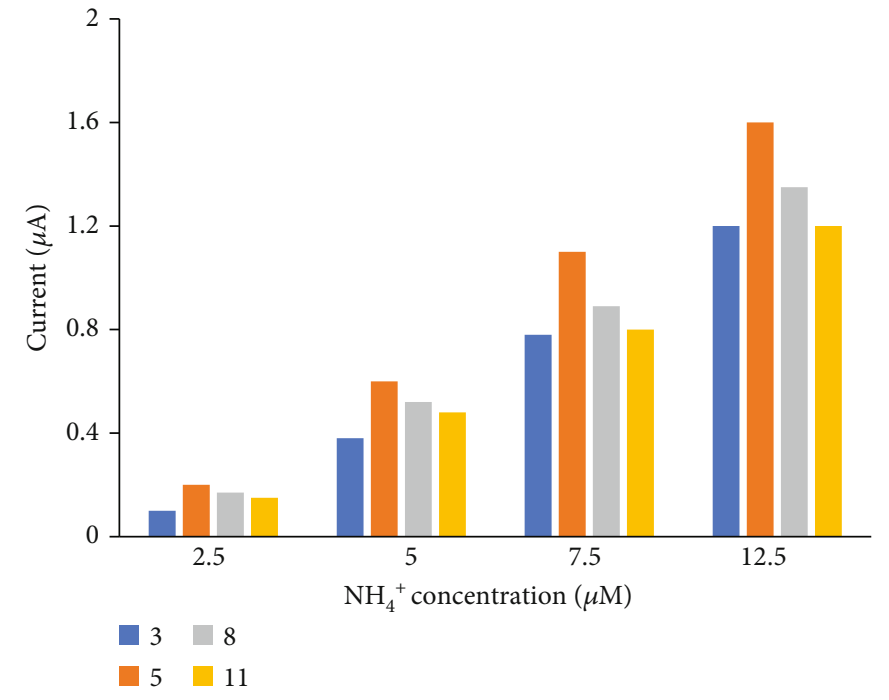

(a)

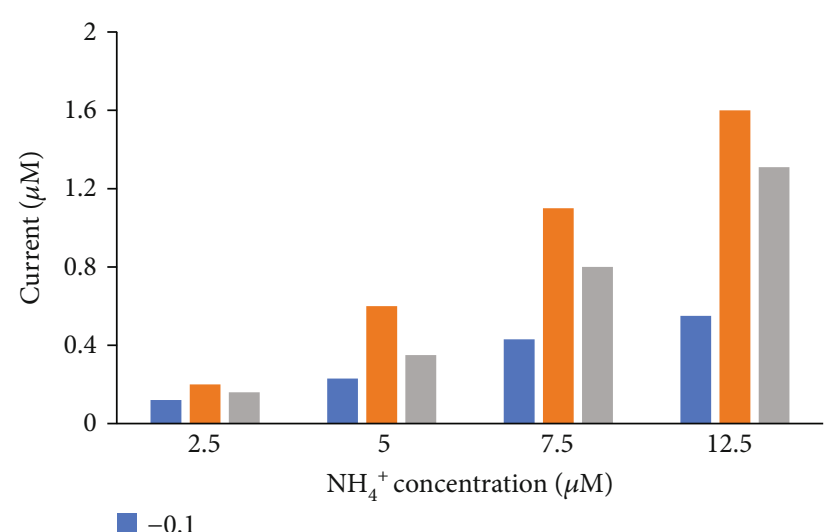

$-0.2$

$-0.3$

Figure 4: The current of the ammonium sensor vs. the concentration of $\mathrm{NH}_{4}^{+}$at (a) different electrodeposition cycles and (b) different constant potentials.

shows the linear sweep voltammograms of the PANI/Nf/Pt electrode in the buffer solution $\mathrm{pH} 7$ containing different concentrations of $\mathrm{NH}_{4}^{+}$. An increased cathodic current at about $-0.2 \mathrm{~V}$ was observed. The transient current responses of various electrodes to successive addition of $2.5 \mu \mathrm{M} \mathrm{NH} \mathrm{NH}_{4}^{+}$ in chronoamperometric experiments at a constant potential of $-0.2 \mathrm{~V}$ are displayed in Figure 3. No cathodic current was seen under ammonium addition at the $\mathrm{Nf} / \mathrm{Pt}$ and PANI/Pt electrodes (amperograms a and b). However, at the $\mathrm{PANI} / \mathrm{Nf} / \mathrm{Pt}$ (amperogram c) electrode, a transient reduction current was observed, proportional to the concentration of ammonium ion in the solution.

The effect of incorporation of MWCNT in the PANI-Nf film was also investigated. A comparison of amperograms $\mathrm{C}$ and $\mathrm{d}$ confirms the higher sensitivity of the PANI/CNT/Nf nanocomposite than the PANI/Nf film. As described by other authors in the literature $[32,33]$, the functional group on the MWCNT surface in $\mathrm{pH} 7$ is deprotonated to form the negative surface charge. This deprotonization may cause an increase in diffusing and movement of cations in the nanocomposite film and, therefore, enhance the current.

Different parameters were optimized by chronoamperometry to obtain the best sensitivity for ammonium ion detection. As shown in Figure 4(a), the sensitivity depends on the number of cycles used for electrodeposition of the PANI film because PANI is indeed the transducer of the sensor. The sensitivity increased up to five cycles, but the responses diminished at higher cycles, probably due to increased film thickness.

Applied working potentials were changed in the range of -0.1 to $-0.3 \mathrm{~V}$ to determine the optimal polarization voltage for amperometric detection of ammonium ion with the current maximum found at $-0.2 \mathrm{~V}$ (Figure $4(\mathrm{~b})$ ).

The SEM technique was used to characterize the morphology of the nanocomposite electrodeposited on the sur-

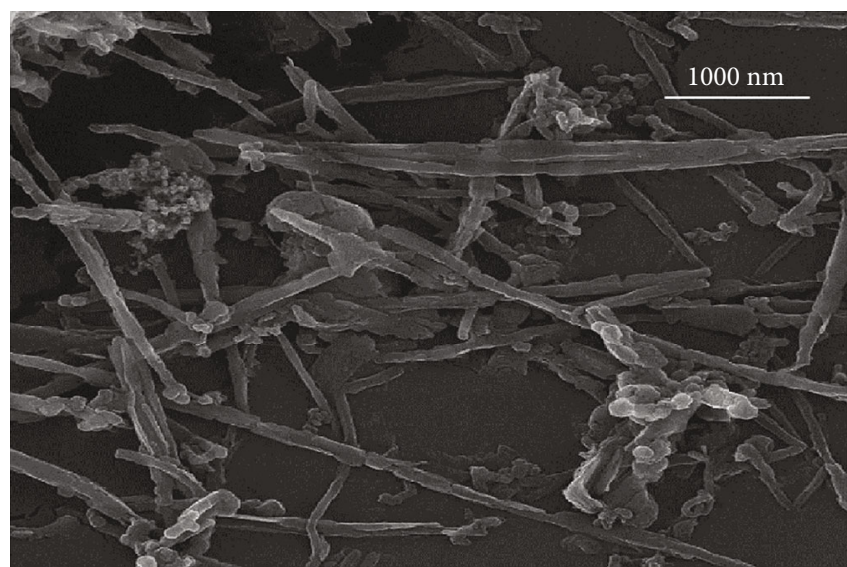

FIgURE 5: SEM image of the PANI/MWCNT/Nafion nanocomposite.

face of the Pt electrode. A typical SEM image is shown in Figure 5. It was evident that after the electrodeposition of the PANI film on the MWCNT-modified electrode, the majority of MWCNTs have been entrapped in the PANI film.

Figure 6 shows a typical FT-IR ATR spectrum of pure polyaniline and the nanocomposite on the surface of a platinum electrode. The spectra of activated MWCNT and Nafion were also presented. The infrared bands are in agreement with those given in the literature for PANI $[24,25]$. The two vibrations of pure polyaniline at 1288 and $1244 \mathrm{~cm}^{-1}$ are assigned to the stretching of the $\mathrm{C}-\mathrm{N}$ bonds of the aromatic amine. The band at $1139 \mathrm{~cm}^{-1}$ is due to a vibrational mode of benzenoid- $\mathrm{NH}^{+}=$quinoid [26], which shows that the polyaniline films formed by electropolymerization are in the emeraldine form. The bands at 1566 and $1491 \mathrm{~cm}^{-1}$ are assignable to the ring stretching vibration of quinoid and benzenoid. It is observed in the FTIR spectra of the 


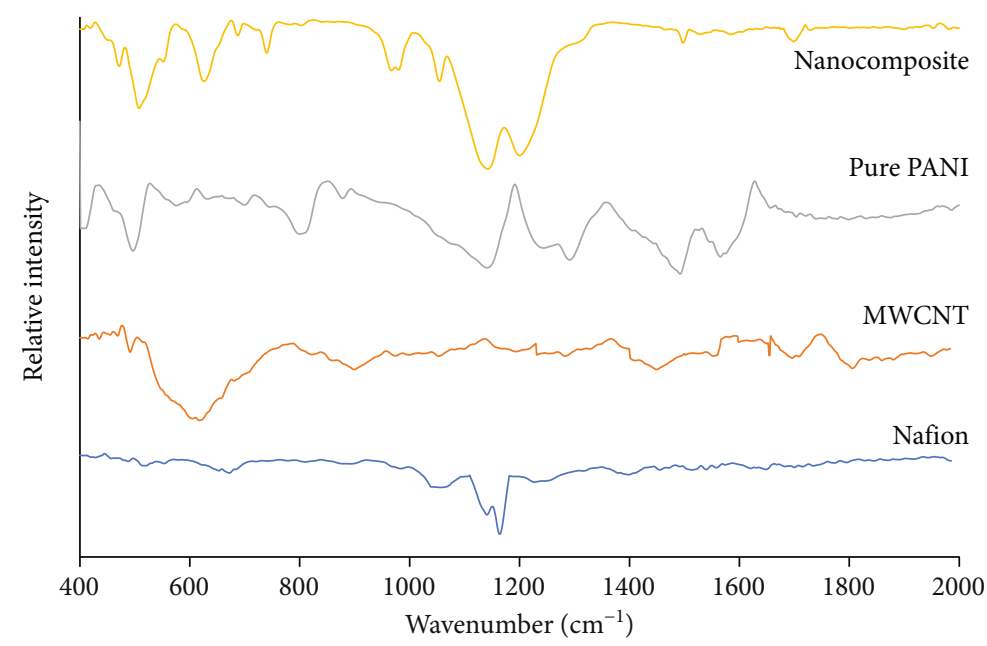

FIGURE 6: FT-IR ATR spectra of Nafion, MWCNT, polyaniline, and the nanocomposite.

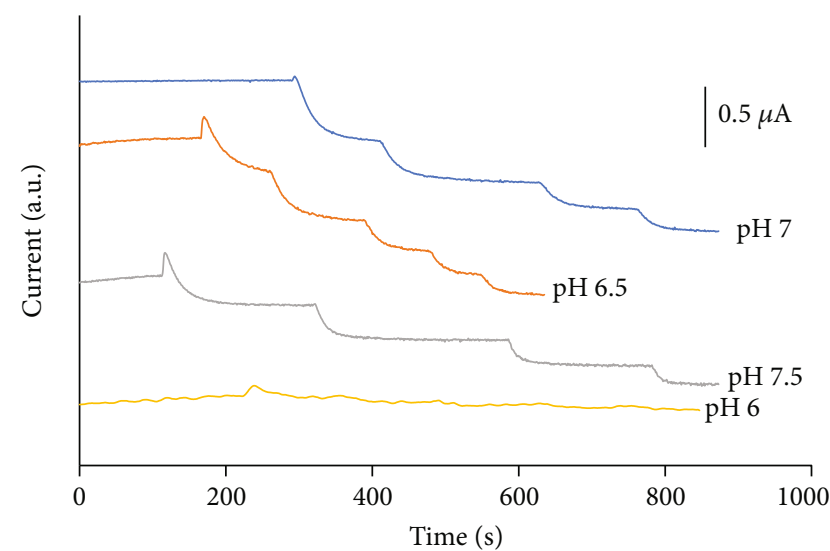

(a)

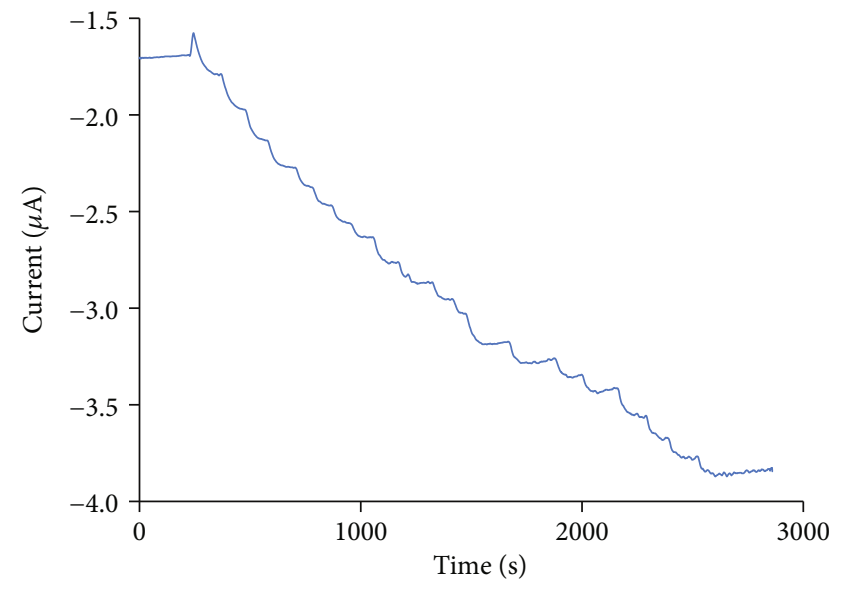

(b)

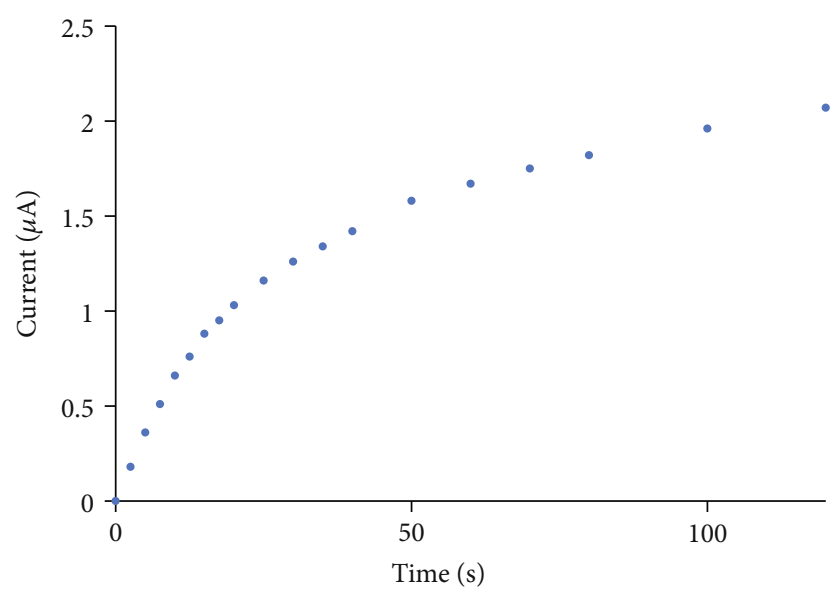

(c)

FIgURE 7: (a) Response current-time curves of the ASNase/PANI/MWCNT/Nf/Pt biosensor at successive addition of $5 \mu \mathrm{M} \mathrm{NH} 4$ in different phosphate buffer solutions, (b) amperometric response curve of the L-Asp biosensor in $0.05 \mathrm{M}$ PBS to successive additions of L-Asp at an applied potential of -0.2 , and (c) a plot of current vs. the concentrations of L-Asp in the range of 0 to $180 \mu \mathrm{M}$.

nanocomposite that polyaniline interacts with MWCNTs and causes redshift of the bands of polyaniline. This effect was previously reported [27]. The band at $1710 \mathrm{~cm}^{-1}$ in
MWCNT spectra is due to the $\mathrm{C}=\mathrm{O}$ stretching frequency, indicating the presence of the carboxylic group created during the activation of MWCNTs. This band also appears in 
TABLE 1: Characteristic of electrochemical L-Asp biosensors reported.

\begin{tabular}{lcccc}
\hline Method & Dynamic range $(\mathrm{M})$ & Response time & Detection limit $(\mu \mathrm{M})$ & References \\
\hline Potentionmetric & $7 \times 10^{-5}-10^{-2}$ & $10 \mathrm{~min}$ & 60 & {$[30]$} \\
Potentionmetric & $1.6 \times 10^{-5}-1.5 \times 10^{-3}$ & $5 \mathrm{~min}$ & - & {$[31]$} \\
Amperometric & $(2-20) \times 10^{-6}$ & - & 0.14 & {$[29]$} \\
Amperometric & $(1-180) \times 10^{-6}$ & $30 \mathrm{~s}$ & This work \\
\hline
\end{tabular}

TABLE 2: The concentration of L-Asp in the blood sample of a patient with acute lymphoblastic leukemia.

\begin{tabular}{lccc}
\hline Sample & $\begin{array}{c}\text { L-Asp }(\mu \mathrm{M}) \\
\text { biosensor }(n=3)\end{array}$ & $\begin{array}{c}\text { L-Asp }(\mu \mathrm{M}) \text { concentration measured by the reference } \\
\text { method }(n=3)\end{array}$ & ${ }^{\mathrm{a}} t_{\text {exp }}{ }^{\mathrm{a}} F_{\text {exp }}$ \\
\hline $\begin{array}{l}\text { Before L-ASNase } \\
\text { injection }\end{array}$ & $64.53 \pm 3.81$ & $61.97 \pm 1.03$ & 1.11 \\
$\begin{array}{l}\text { After L-ASNase } \\
\text { injection }\end{array}$ & $7.96 \pm 0.47$ & $8.33 \pm 0.099$ & 13.6 \\
\hline
\end{tabular}

${ }^{a} t_{\text {exp }}$ and $F_{\text {exp }}$ show the experimental Student $t$ and experimental $F$ values, respectively $\left(t_{0.05}-2.78\right.$ and $\left.F_{0.05}(2,2)-39.25\right)$.

the nanocomposite spectrum at $1700 \mathrm{~cm}^{-1}$ because the polyaniline peaks are absent in this region. In Nafion spectra, the band at $1056 \mathrm{~cm}^{-1}$ and $1154 \mathrm{~cm}^{-1}$ is attributed to $\mathrm{S}=\mathrm{O}$ and $\mathrm{CF}_{2}$ stretching vibrations [28], respectively. But these bands are not observed in the nanocomposite spectra because they are masked by stronger PANI peaks.

\subsection{Electrochemical Performances of the L-Asp} Nanobiosensor. The L-Asp nanobiosensor (ASNase/PANI/MWCNT/Nf/Pt) was prepared by immobilization of LASNase on the PANI/MWCNT/Nf/Pt electrode. The influence of $\mathrm{pH}$ on the performance of the nanobiosensor was studied, and the results are shown in Figure 7(a). The highest current was found at $\mathrm{pH} 7.0$, and it was employed throughout the study.

Figure 7(b) displays the amperometric response of the ASNase/PANI/MWCNT/Nf/Pt nanobiosensor to the sequential increment of L-Asp concentration at a constant potential of $-0.2 \mathrm{~V}$.

A plot of the current versus the concentration of L-Asp is shown in Figure $7(\mathrm{c})$. A dynamic concentration range was observed from 0 to $180 \mu \mathrm{M}$. The current intensity of the biosensor toward L-Asp increased linearly over the concentration range of 0 to $15 \mu \mathrm{M}$. The equation for the calibration line was $I(\mu \mathrm{A})=0.0586 C(\mu \mathrm{M})+0.0393$ with $R^{2}=0.990$, where $C$ in the above equation is the L-Asp concentration in $\mu \mathrm{M}$ and $I$ is the current in $\mu \mathrm{A}$. The sensitivity of the biosensor was $0.829 \mu \mathrm{A} \mu \mathrm{M}^{-1} \mathrm{~cm}^{-2}$, and the limit of detection at a signal-to-noise ratio of 3 was estimated as $0.14 \mu \mathrm{M}$. Stepwise growth of reduction current was observed with increasing amounts of L-Asp in PBS, and the current reached a steady-state level of less than $30 \mathrm{~s}$ (response time). The reproducibility (RSD) of this sensor was $2.9 \%(n=5)$ to determine $10 \mu \mathrm{M} \mathrm{L}$-Asp. The performance of the proposed nanobiosensor was compared with other L-Asp biosensors in Table 1. Its response time and detection limit are lower than other electrochemical biosensors that have ever been reported [34].

The apparent Michaelis-Menten constant $\left(K_{m}^{\mathrm{app}}\right)$ indicates the enzyme-substrate affinity for the biosensors. The electrochemical version of the Lineweaver-Burk equation can be applied to calculate $K_{m}^{\text {app }}$ :

$$
\frac{1}{i_{\mathrm{ss}}}=\frac{1}{i_{\max }}+\frac{K_{m}^{\mathrm{app}}}{i_{\max } c}
$$

where $i_{\mathrm{ss}}$ is the steady-state current after adding substrate, $C$ is the bulk concentration of the substrate, and $i_{\max }$ is the maximum current measured under saturated substrate condition [35]. The calculated $K_{m}^{\mathrm{app}}$ of the L-Asp biosensor is $36.2 \mathrm{mM}$.

3.4. Real Sample Analysis. The biosensor was also tested for the L-Asp quantification in serum samples of the patient with leukemia before and after treatment with L-ASNase using the standard addition method. The presence of endogenous ammonium in the samples interferes with these determinations since the response measured corresponds to the sum of the ammonium generated enzymatically and present in the sample. Therefore, the latter was additionally measured in serum samples with the MWCNT/PANI/Nf/Pt electrode, and the concentration of L-Asp was calculated as a difference between the signals of PANI/MWCNT/Nf/Pt- and ASNase/PANI/MWCNT/Nf/Pt-based sensors (Table 2). The results of a reference method (explained in the experimental part) are also given in Table 2. The $t$-test was applied to compare the results of the two methods. As seen from Table 2, the proposed biosensor results agree well with those obtained using a reference method.

\section{Conclusion}

The MWCNT/PANI/Nf nanocomposite was utilized to fabricate a new amperometric L-Asp nanobiosensor that exhibited high sensitivity and selectivity. The nanobiosensor was specifically and successfully used to monitor L-Asp in the patient's blood with acute lymphoblastic leukemia. One can expect that the proposed biosensor has the potential to be used as a point-of-care testing device. However, it will be the subject of further investigation in the future. The 
proposed nanocomposite might be considered for a wide range of biosensors based on the detection of ammonium ions. This novel nanobiosensor is a rapid, accurate, well-handled, portable vessel for in situ detection of the serum concentration anticancer agent L-asparaginase in human blood during chemotherapy. This approach helps make sure that the patient receives the optimal dose of the drug with the minimum side effect.

\section{Data Availability}

All data used to support the findings of this study are included within the article.

\section{Conflicts of Interest}

The authors declare that they have no conflicts of interest.

\section{Acknowledgments}

This study has been performed according to grant no. 8478 funded by vice chancellery for research affairs, Shiraz University of Medical Sciences. We would also like to express our gratitude to Prof. Afsaneh Safavi, Department of Chemistry, Shiraz University, for allowing us to use her lab facilities and electrochemical workstation.

\section{References}

[1] M. J. Raee, A. Ebrahiminezhad, A. Gholami, M. B. Ghoshoon, and Y. Ghasemi, "Magnetic immobilization of recombinant $E$. coli producing extracellular asparaginase: an effective way to intensify downstream process," Separation Science and Technology, vol. 53, no. 9, pp. 1397-1404, 2018.

[2] K. Schilling, M. C. Amstalden, L. Meinel, and U. Holzgrabe, "Impurity profiling of l-asparagine monohydrate by ion pair chromatography applying low wavelength UV detection," Journal of pharmaceutical and biomedical analysis, vol. 131, pp. 202-207, 2016.

[3] Y. Zhang, Y. Wang, R. Wang et al., "Personalized nanomedicine: a rapid, sensitive, and selective UV-vis spectrophotometry method for the quantification of nanostructured PEGasparaginase activity in children's plasma," International Journal of Nanomedicine, vol. Volume 13, pp. 6337-6344, 2018.

[4] A. Magri, M. F. Soler, A. M. Lopes et al., "A critical analysis of L-asparaginase activity quantification methods-colorimetric methods versus high-performance liquid chromatography," Analytical and bioanalytical chemistry, vol. 410, no. 27, pp. 6985-6990, 2018.

[5] A. Gholami, S. Shahin, M. Mohkam, N. Nezafat, and Y. Ghasemi, "Cloning, characterization and bioinformatics analysis of novel cytosine deaminase from Escherichia coli AGH09," International Journal of Peptide Research and Therapeutics, vol. 21, no. 3, pp. 365-374, 2015.

[6] S. A. Hashemi, N. G. G. Behbahan, S. Bahrani et al., "Ultrasensitive viral glycoprotein detection NanoSystem toward accurate tracing SARS-CoV-2 in biological/non-biological media," Biosensors and Bioelectronics, vol. 171, p. 112731, 2021.

[7] M. J. Raee, A. Ebrahiminezhad, M. B. Ghoshoon, A. Gholami, and Y. Ghasemi, "Synthesis and characterization of L-lysin coated iron oxide nanoparticles as appropriate choices for cell immobilization and magnetic separation," Nanoscience \& Nanotechnology-Asia, vol. 9, no. 4, pp. 462-466, 2019.

[8] H. N. Jayaram, D. A. Cooney, and C. Y. Huang, "Interaction between L-aspartic acid and L-asparaginase from Escherichia coli: binding and inhibition studies," Journal of enzyme inhibition, vol. 1, no. 2, pp. 151-161, 1986.

[9] S. M. Mousavi, M. Zarei, S. A. Hashemi et al., "Gold nanostarsdiagnosis, bioimaging and biomedical applications," Drug metabolism reviews, vol. 52, no. 2, pp. 299-318, 2020.

[10] W. Hao, G. Das, and H. H. Yoon, "Fabrication of an amperometric urea biosensor using urease and metal catalysts immobilized by a polyion complex," Journal of Electroanalytical Chemistry, vol. 747, pp. 143-148, 2015.

[11] S. Dzyadevych, V. Arkhypova, A. Soldatkin, A. El'Skaya, C. Martelet, and N. Jaffrezic-Renault, "Biocapteurs enzymatiques a transduction amperometrique: passe, present, futur," IRBM, vol. 29, no. 2-3, pp. 171-180, 2008.

[12] C.-H. Liu, K.-T. Liao, and H.-J. Huang, “Amperometric immunosensors based on protein A coupled polyaniline-perfluorosulfonated ionomer composite electrodes," Analytical chemistry., vol. 72, no. 13, pp. 2925-2929, 2000.

[13] B. Lakard, "Electrochemical biosensors based on conducting polymers: a review," Applied Sciences, vol. 10, no. 18, p. 6614, 2020.

[14] A. D. Chowdhury, R. Gangopadhyay, and A. De, "Highly sensitive electrochemical biosensor for glucose, DNA and protein using gold-polyaniline nanocomposites as a common matrix," Sensors and Actuators B: Chemical., vol. 190, pp. 348-356, 2014.

[15] A. Wang, X. Ma, Y. Ye et al., "A simple and convenient aptasensor for protein using an electronic balance as a readout," Analytical Chemistry., vol. 90, no. 2, pp. 1087-1091, 2018.

[16] B. Zhang, Y. He, B. Liu, and D. Tang, "Nickel-functionalized reduced graphene oxide with polyaniline for non-enzymatic glucose sensing," Microchimica Acta, vol. 182, no. 3-4, pp. 625-631, 2015.

[17] Y. Cui, H. Chen, D. Tang, H. Yang, and G. Chen, “Au(iii)-promoted polyaniline gold nanospheres with electrocatalytic recycling of self-produced reactants for signal amplification," Chemical Communications., vol. 48, no. 83, pp. 10307-10309, 2012.

[18] J. Shu, Z. Qiu, S. Lv, K. Zhang, and D. Tang, "Cu2+-doped $\mathrm{SnO} 2$ nanograin/polypyrrole nanospheres with synergic enhanced properties for ultrasensitive room-temperature H2S gas sensing," Analytical Chemistry, vol. 89, no. 20, pp. 11135-11142, 2017.

[19] J. Shu, Z. Qiu, and D. Tang, "Self-referenced smartphone imaging for visual screening of $\mathrm{H} 2 \mathrm{~S}$ using CuxO-polypyrrole conductive aerogel doped with graphene oxide framework," Analytical Chemistry., vol. 90, no. 16, pp. 9691-9694, 2018.

[20] G. Cai, Z. Yu, and D. Tang, "Actuating photoelectrochemical sensing sensitivity coupling core-core-shell $\mathrm{Fe}_{3} \mathrm{O}_{4} @[$ email protected $]_{2}$ with molecularly imprinted polypyrrole," Talanta, vol. 219, p. 121341, 2020.

[21] M. P. Massafera and S. I. C. de Torresi, "Urea amperometric biosensors based on a multifunctional bipolymeric layer: comparing enzyme immobilization methods," Sensors and Actuators B: Chemical, vol. 137, no. 2, pp. 476-482, 2009.

[22] W. Cho, F. Liu, A. Hendrix, T. Asrat, V. Connaughton, and A. G. Zestos, "Timed electrodeposition of PEDOT: Nafion 
onto carbon fiber-microelectrodes enhances dopamine detection in zebrafish retina," Journal of The Electrochemical Society, vol. 167, no. 11, p. 115501, 2020.

[23] N. Ebrahimi, S. Gharibi, M. Ghoshoon et al., "Selective isolation and identification of arginine degrading bacteria; the optimized arginine deaminase production by Enterobacter sp. sgn 1 as a new source of this potentially anti-tumor enzyme," Journal of Applied Pharmaceutical Science, vol. 6, no. 9, pp. 093-101, 2016.

[24] J. Shu and D. Tang, "Recent advances in photoelectrochemical sensing: from engineered photoactive materials to sensing devices and detection modes," Analytical Chemistry., vol. 92, no. 1, pp. 363-377, 2020.

[25] F. Emadi, A. Emadi, and A. Gholami, "A comprehensive insight towards pharmaceutical aspects of graphene nanosheets," Current pharmaceutical biotechnology., vol. 21, no. 11, pp. 1016-1027, 2020.

[26] H. Sa, S. M. Mousavi, A. Gholami, S. Mazraedoost, W.H. Chiang, and O. Arjmand, "Precise blood glucose sensing by nitrogen-doped graphene quantum dots for tight-control of diabetes," Journal of Sensors, 2021.

[27] D. Maity, M. Manoharan, and R. T. Rajendra Kumar, "Development of the PANI/MWCNT nanocomposite-based fluorescent sensor for selective detection of aqueous ammonia," ACS Omega, vol. 5, no. 15, pp. 8414-8422, 2020.

[28] B. K. Shrestha, R. Ahmad, S. Shrestha, C. H. Park, and C. S. Kim, "Globular shaped polypyrrole doped well-dispersed functionalized multiwall carbon nanotubes/Nafion composite for enzymatic glucose biosensor application," Scientific Reports, vol. 7, no. 1, p. 16191, 2017.

[29] V. H. Nguyen and J.-J. Shim, "Green synthesis and characterization of carbon nanotubes/polyaniline nanocomposites," Journal of Spectroscopy, vol. 2015, Article ID 297804, 2015.

[30] A. Kumar, V. Kumar, and K. Awasthi, "Polyaniline-carbon nanotube composites: preparation methods, properties, and applications," Polymer-Plastics Technology and Engineering, vol. 57, no. 2, pp. 70-97, 2018.

[31] D. Ragupathy, A. Gopalan, K.-W. Kim, and K. P. Lee, "Synthesis and characterization of polyaniline grafted multiwalled carbon nanotube loaded Nafion-silica nanocomposite membrane," Journal of nanoscience and nanotechnology., vol. 11, no. 1, pp. 747-750, 2011.

[32] X. Ren, J. Li, X. Tan, and X. Wang, "Comparative study of graphene oxide, activated carbon and carbon nanotubes as adsorbents for copper decontamination," Dalton Transactions., vol. 42, no. 15, pp. 5266-5274, 2013.

[33] F. Qu, M. Yang, J. Jiang, G. Shen, and R. Yu, “Amperometric biosensor for choline based on layer-by-layer assembled functionalized carbon nanotube and polyaniline multilayer film," Analytical Biochemistry, vol. 344, no. 1, pp. 108-114, 2005.

[34] A. Erdogan, S. Koytepe, B. Ates, I. Yilmaz, and T. Seckin, "Preparation of the L-asparaginase-based biosensor with polyimide membrane electrode for monitoring L-asparagine levels in leukemia," International Journal of Polymeric Materials and Polymeric Biomaterials., vol. 63, no. 17, pp. 909-917, 2014.

[35] A. Safavi and F. Farjami, "Electrodeposition of gold-platinum alloy nanoparticles on ionic liquid-chitosan composite film and its application in fabricating an amperometric cholesterol biosensor," Biosensors and Bioelectronics., vol. 26, no. 5, pp. 2547-2552, 2011. 